\title{
Automated recognition and counting of the immunoreactive neuroendocrine cells in chronic gastritis (the preliminary study)
}

\author{
Tomasz Markiewicz ${ }^{1,2}$, Cezary Jochymski' ${ }^{1}$, Janina Slodkowska ${ }^{1}$, Wojeiech Kozlowski ${ }^{1}$ \\ ${ }^{1}$ Department of Pathology, Military Institute of Medicine, Poland \\ ${ }^{2}$ Warsaw University of Technology, Poland
}

\begin{abstract}
The paper presents the designed software CAMI (Computerized Analysis of Microscopic Images) for a digital reconstruction of the diversiform glands seen in chronic inflammatory gastric mucosa, and for automated recognition and quantization of the immunoreactive neuroendocrine (NE) cells appearing within mucosal glands. Digital reconstruction of the individual gastric gland is difficult due to variable shapes of the glandular cross-sections. Fifteen gastric biopsy specimens representing chronic gastritis were stained routinely with $\mathrm{H}+\mathrm{E}$ and immunohistochemically with 3 NE markers: Chromogranin A, Somatostatin and Serotonin. Two expert pathologists counted manually the NE cells with the light microscope in 4 types of glandular cross-sections: round, short- oblique, long- oblique and longitudinal. The automated counting of the NE cells was performed on the digital images presenting the same microscopic areas which were selected for the manual reading. The first step of image analysis was concerned to the cell extraction and recognition of the cytoplasmic immunoreactivity. The unstained nuclei of the NE cells were spotted by the sequential thresholding algorithm combined with the artificial neural network of SupportlVector Machine (SVM) type. The second step of image analysis comprised reconstruction of the glands. The presumed shape of each gastric gland was defined by the cellular lining of viewed glandular cross-section. The designed algorithm for gland reconstruction was based on the cell masks. The third step of analysis dealt the cell counting. Every recognized gland with the face cells was used for the NE cell evaluation. The results of the automated quantization compared with manual counting results for the number of NE cells showed high concordance in 3 types of glandular cross-sections: round, short- and long- oblique. A difference noticed in the results of the longitudinal glands should be verified in the extended study. The designed software CAMI is more adequate for the gland recognition with an discontinuous gland face seen in the immunohistochemical digital images, which appear to be a difficult problem for the accurate automated analysis of the cellular component of glands.
\end{abstract}

Keywords: immunoreactive neuroendocrine cell, chronic gastritis, cell recognition, cell extraction, Support Vector Machine.

\section{Introduction}

Endoscopic evaluation and mucosa biopsy analysis play important roles in the patients management with inflammatory gastric disorders. Gastric neuroendocrine (NE) cells comprise about $1 \%$ of the volume of the oxyntic mucosa, and represent several cell types like: enterochromaffin-like cells (ECL), G cells, and D cells, with their secretory products including chromogranin, histamine, gastrin, and somatostatin. Variable numbers and various subtypes of the NE cells have

Correspondence: T. Markiewicz, Warsaw University of Technology, Koszykowa Str. 75, 00-662 Warszawa, Poland; e-mail: markiewj@wp.pl been described in association with different types of chronic gastritis [1-6]. The endocrine cell population of the stomach may occur as the hyperplastic lesions usually in the setting of chronic atrophic gastritis. In atrophic body gastritis chronic hypergastrinaemia stimulates ECL cell proliferation with development of cell hyperplasia, dysplasia and possibly type-1 gastric tumour $[7,8]$. Neuroendocrine proliferation of gastric mucosa may be also an indirect effect of modern drugs suppressing acid secretion (the post-pharmacotherapeutic hypo- or achlorhydric states). To the present there is a lack of accepted evidence on the participation of NE cells in the stomach inflammation pathology.

There is a wide range of methods used for evaluation of NE cells density in the histological slides 

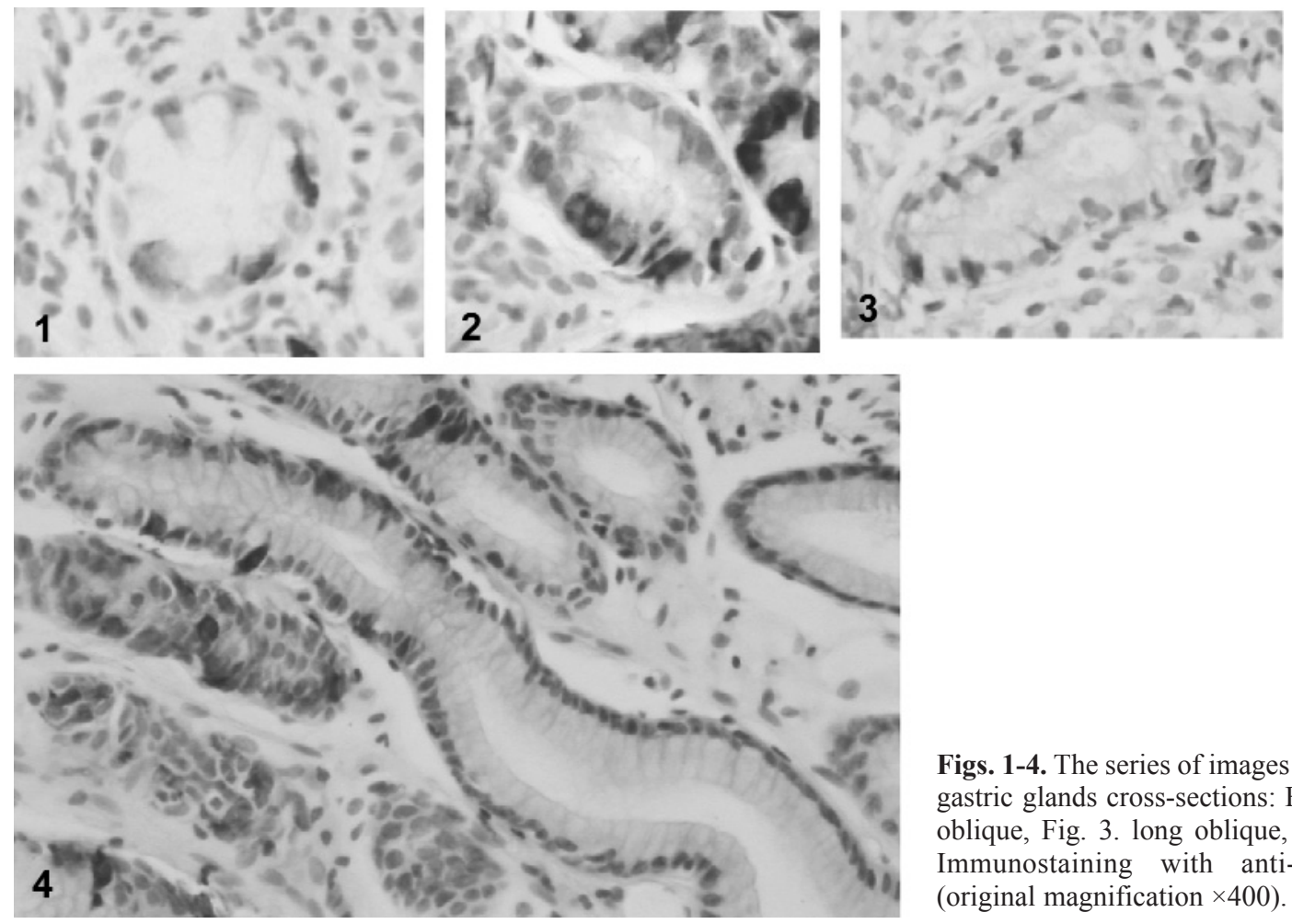

Figs. 1-4. The series of images present 4 categories of the gastric glands cross-sections: Fig. 1. round. Fig. 2. short oblique, Fig. 3. long oblique, Fig. 4. longitudinal type. Immunostaining with anti-chromogranin antibody (original magnification $\times 400$ ).

$[3-5,9]$. Some researchers counted the endocrine cells in a few microscopic fields, at magnification $\times 200$ and presented results as the mean number of cells per one microscopic field [3]. Other investigators measured the number of NE cells per $\mathrm{mm}^{2}$ corresponding to the area of the mucosal glandular epithelium or per one gland $[4,5,9]$. More recently we found the data reporting the percentage of immunoreactive cells in relation to the total number of all stained cells appearing in any random microscopic field or as a number of neuroendocrine cells in a few microscopic fields [10,11]. Lack of consensus in quantitative methods used for the NE cells counting in inflamed gastric mucosa might result from the shape diversity of the gastric glands and the difficulties in their reconstruction on the digital images. In our previous study with the light microscopy, the NE cells were counted by semi-quantitative method in 4 types of gastric glands cross-sections: round, short- and long-oblique, and longitudinal. The NE cells were counted at least in 5 glandular cross-sections of each type and the final results were expressed as a sum of mean numbers of NE cells for all types of glandular cross-sections per one slide [6].

The automated computerized systems able to recognize and count the NE cells are of the great aids for the pathologists in the objective and precise quantisation of the NE cells. Our software CAMI (Computerized Analysis of Microscopic Images) was designed for recognition and counting of the immunoreactive NE cells within reconstructed cross sections of the gas- tric glands. Digital reconstruction of the individual gastric gland is not easy task because of variable shapes of the glandular cross-sections (ranging from round to longitudinal). It results from the glandular ramifications and various cutting planes of the glands within gastric mucosa. Some specific glandular shapes are related to the anatomic part of the stomach (pyloric versus oxyntic area). Our study was focused only on the pyloric area glands which are easy to recognize and reconstruct by the computerized system in the model of chronic gastritis.

We aimed to design an algorithm for a digital reconstruction of the diversiform glands seen in chronic inflammatory gastric mucosa, and build the algorithm for automated recognition and quantization of the immunoreactive NE cells appearing within diversiform mucosal glands in a model of chronic gastritis.

\section{Materials and methods}

Eighteen gastric biopsy specimens obtained from the patients of the MIHS (in Warsaw, Poland) entered the study. In the microscopic evaluation - 15 specimens represented chronic gastritis (ChG), 2 - superficial chronic gastritis ( $\mathrm{SChG);} \mathrm{in} \mathrm{one} \mathrm{case} \mathrm{the}$ gastric biopsy did not reveal any abnormality. The gastric mucosa sections were stained routinely with $\mathrm{H}+\mathrm{E}$ and immunohistochemically with 3 NE markers: Chromogranin A, Somatostatin and Serotonin, according to the manufacturer recommendations (Dako, Denmark). We used the following dilutions of antibodies: Monoclonal Mouse Anti-Human Chromogranin A, (clone DAK-A3, code M 0869) - 1:100, Monoclonal Mouse Anti-Serotonin (clone 5HT-H209, code M 0758) - 1:50, Rabbit Polyclonal Anti-Human 
a

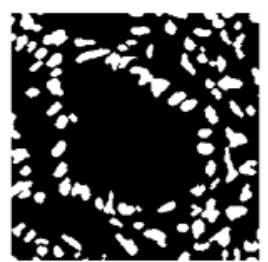

b

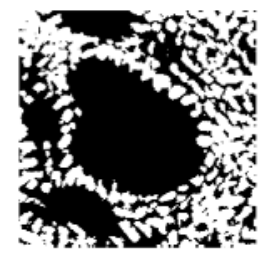

c

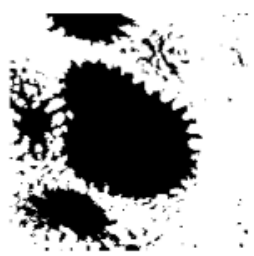

$\mathrm{d}$

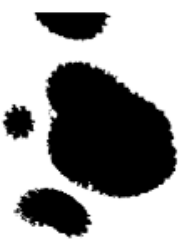

Fig. 5a-d. The images present the steps of a gland shape reconstruction done by the repeated HMT application on the same image: a. an initial mask of the nuclei, b. the mask after one HMT application, c. result of double HMT application, d. quadruple HMT applications.

Somatostatin (code A0566 ); and Dako EnVision as the visualization system. The NE cells were counted at magnification $\times 400$; separately for each NE marker; in 10 selected microscopic fields which showed the highest density of NE cells.

Two expert pathologists (specialised in gastric pathology) examined the specimens with the light microscope and counted manually the number of NE cells. The morphological criteria for the gastritis agreed with the modified Whitehead classification of the gastritis [6]. The gastric glands were classified according to their cross-section shapes as follows: 1/ round, 2/ short oblique, 3 / long oblique and 4/ longitudinal cross-sections gland. The NE cells were counted at least in 5 glands of each type in every glass slide and the final results were expressed as a sum of NE cells numbers for all gland types [6].

The automated counting of the NE cells was performed on the digital images presenting the same microscopic areas which were indicated by expert pathologist for the manual reading. For the images acquisition the microscope Olympus BX50 with C3030 Camedia system was used; magnification $\times 400$; resolution $768 \times 576$ pixels. Maximum five glands of each category in the view fields were digitized (Fig. 1-4).

The method of gland reconstruction based on the immunoreactive cells recognition and their counting within a gland cross-section. We used the following criteria for the recognition of the immunopositive cell: 1/ well visible blue nucleus and brown rounded cytoplasmic "body" (product of immunoreaction) bordered its nucleus, or 2/ the cell with brown cytoplasm "body" of a size comparable to the nucleus of an adequate cell.

The first step of image analysis was concerned to the cell extraction and recognition of the cytoplasmic immunoreactivity. For the cell extraction we used the special algorithm previously designed and successfully applied for the Index Ki-67 calculation in neuroblastomas [18]. The recognition of cytoplasmic immunoreactivity was based on the algorithm adapted from our previous study on bone marrow [19].

The second step of image analysis comprised reconstruction of the glands. The presumed shape of each gastric gland was defined by the cellular lining of viewed glandular cross-section. The designed algorithm for gland reconstruction was based on the cell masks. The masks of the immunonegative and immunopositive cells helped in reconstruction of the glandular cross-section. The cytoplasmic immunoreactivity was specified by brown colour within transparent (or pale blue) cytoplasmic compartment, the nuclei were dark blue colour. Because the contours of the immunonegative cells were not visible, we used the nuclear appearance as a base for the digital gland reconstruction. We assumed that the gland face was formed by the outlines of the cell faces seen as the blue rims bounded together. For recognition of the complete gland outlining the computerized system had to fill the spaces between the nuclei of the glandular cells. The process of gland reconstruction was covered by the Hit-or-Miss (HMT) morphological operation with anisotropic structure element (SE) of a line shape 35 pixels (SE length) $[20,21]$. The based pixel of SE was a center point on the line shape, the SE foreground was formed by 3 last pixels at both ends; the rest of pixels of the line shape formed a background. The optimal SE structure was picked out from the series of tests in regards to image resolution and magnification. Because of the nuclear multipolarity within a gland contour, the Hit-or-Miss operation had to be done in the series of SE rotation (every $5^{\circ}$ in the scale from $0^{\circ}$ to $175^{\circ}$ ). The Hit-or-Miss operation could perform only the masks for areas in-between stained closest nuclei. In order to obtain the mask of the whole glandular region the HMT operation had to be performed twice with 35 pixels long SE and twice with 23 pixels long SE, subsequently. By addition of these masks to the masks obtained in the first step of algorithm analysis, a new mask was created. This mask covered the luminal cellular lining of gland contour. This step of algorithm analysis is illustrated by the images on Fig. 5a-d.

The main reason of such programmed gland contour reconstruction is that the original gland lumen cannot be reconstructed by simple closing or dilate operation. These operations allow to recognize the gland with short width, without false cutting one gland shape into several separated glands. The final step of gland recognition that was done by getting any separated area with size higher than 1000 pixels. For any of these objects the cellular contours were detected by a dilate operation of its area with SE in the disk shape and radius equals 8 pixels. After dilation the mask of the gland area covered every cell from this gland face and these cells can be easy ascribed to a proper gland. Each glandular object was measured in its longest and shortest axis for the proper categorization of its shape.

The third step of analysis dealt the cell counting. Every recognized gland with the face cells was used for the NE cell evaluation.

\section{Results}

The process of cell and gland recognition by CAMI software is seen on Fig. 6.

Most of the glands with correct cross-sections were recognized. Six glands were fully extracted with the their face cells. One object created by the software as a gland from the stroma had to be eliminated by a user.

The results of NE cells counting obtained by both methods (automated and manual) are shown only for Chromogranin A staining (Table 1). The number of NE cells seen in the microscopic slides stained with other NE markers: Serotnin and Somatostatin was so small to show the convincing data. Due to a shortage of cases representing superficial chronic gastritis our analysis was focused on the model of chronic gastritis. In the manual reading the mean number of the NE cells in round shape glands was 2.63, in short oblique glands 3.54 , in long oblique -5.19 . In the automated quantitation of NE cells the final results were similar for the same 3 types of glands: $2.37 ; 3.77$ and 6.02 , respec- 


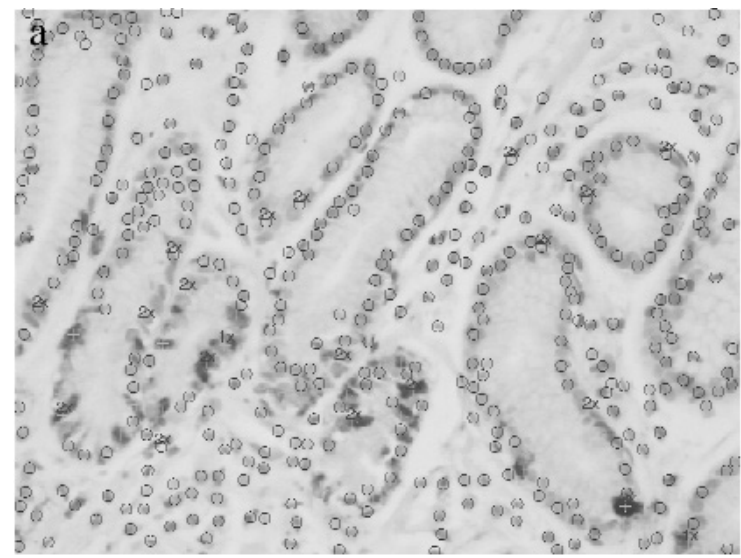

b
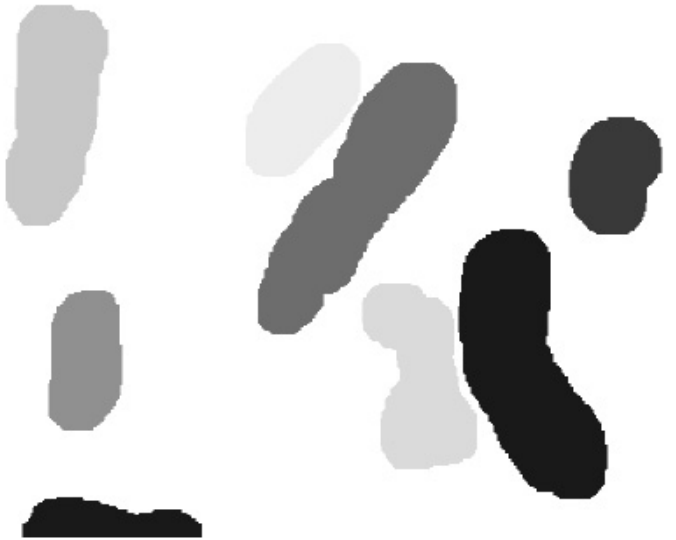

Fig. 6. The process of the computerized recognition of cells and glands on the image with Chromogranin-A immunostaining: a. the image of marked recognized cells, b. the mask of reconstructed glands, c. and $\mathbf{d}$. represent two detected glands with cells on the gland outlining. The immunopositive cells are marked by " $2+$ ", the immunonegative by "o".

Table 1. The results of NE cells counting performed by CAMI and manual reading for Chromogranin immunostained slides.

\begin{tabular}{|c|c|c|c|c|c|c|c|c|c|c|}
\hline \multirow{3}{*}{$\begin{array}{c}\text { Histological } \\
\text { diagnosis }\end{array}$} & \multicolumn{5}{|c|}{$\begin{array}{c}\text { Number of NE cells (mean number) in glands by CAMI } \\
\text { evaluation }\end{array}$} & \multicolumn{5}{|c|}{$\begin{array}{l}\text { Number of NE cells (mean number) in glands by manual } \\
\text { reading }\end{array}$} \\
\hline & \multicolumn{5}{|c|}{ Categories of glands } & \multicolumn{5}{|c|}{ Categories of glands } \\
\hline & A & B & $\mathrm{C}$ & $\mathrm{D}$ & All categories & $\mathrm{A}$ & B & $\mathrm{C}$ & $\mathrm{D}$ & All categories \\
\hline 1. normal & 2 & 3.8 & 3.2 & 5 & 14.0 & 1 & 2.5 & 5.8 & 9 & 18.3 \\
\hline 2. $\mathrm{SChG}$ & 4.2 & 6.4 & 2.8 & 0 & 13.4 & 3.8 & 3.5 & 7.5 & 0 & 14.8 \\
\hline 3. $\mathrm{SChG}$ & 2.5 & 5.8 & 8 & 0 & 16.3 & 2 & 7 & 9.2 & 0 & 18.2 \\
\hline 4. $\mathrm{ChG}$ & 2.5 & 5 & 7.8 & 0 & 15.6 & 3 & 5.2 & 7 & 0 & 15.2 \\
\hline 5. $\mathrm{ChG}$ & 3 & 3 & 8.7 & 3.5 & 18.2 & 3.5 & 4.8 & 5 & 8 & 17.8 \\
\hline 6. $\mathrm{ChG}$ & 3 & 4.7 & 2 & 0 & 9.7 & 1 & 2.5 & 6.8 & 0 & 10.3 \\
\hline 7. $\mathrm{ChG}$ & 3.2 & 2.4 & 4.4 & 0 & 10.0 & 2.4 & 3.8 & 5.7 & 0 & 11.9 \\
\hline 8. $\mathrm{ChG}$ & 2 & 1.7 & 5.8 & 0 & 9.5 & 2 & 4 & 6.8 & 0 & 12.8 \\
\hline 9. $\mathrm{ChG}$ & 1.8 & 4.4 & 7.3 & 0 & 13.5 & 2 & 7.8 & 6.8 & 0 & 13.6 \\
\hline 10. $\mathrm{ChG}$ & 4 & 3.6 & 5.4 & 0 & 13.0 & 3.3 & 3.4 & 7.2 & 0 & 13.9 \\
\hline 11. $\mathrm{ChG}$ & 3.2 & 2.3 & 4 & 0 & 9.5 & 2.2 & 2.6 & 4 & 0 & 8.8 \\
\hline 12. $\mathrm{ChG}$ & 3.4 & 3 & 7.3 & 7 & 20.7 & 2.8 & 4.2 & 7.2 & 8 & 22.2 \\
\hline 13. $\mathrm{ChG}$ & 2 & 1.4 & 4.4 & 0 & 7.8 & 2.3 & 2 & 4.8 & 0 & 9.1 \\
\hline 14. $\mathrm{ChG}$ & 1.6 & 3 & 2.2 & 0 & 6.8 & 1.4 & 2.6 & 3.6 & 0 & 7.6 \\
\hline 15. $\mathrm{ChG}$ & 4 & 3.3 & 4.5 & 0 & 11.8 & 3 & 2.3 & 6.3 & 0 & 11.7 \\
\hline 16. $\mathrm{ChG}$ & 2.2 & 2.3 & 2.6 & 0 & 7.1 & 2.3 & 3.6 & 3.8 & 0 & 9.7 \\
\hline 17. ChG & 1.8 & 4.5 & 8 & 2 & 16.3 & 2 & 2.4 & 6 & 7.8 & 16.2 \\
\hline 18. $\mathrm{ChG}$ & 1 & 3.2 & 5 & 0 & 9.2 & 2.7 & 3.7 & 5 & 0 & 11.3 \\
\hline $\begin{array}{l}\text { Mean } \\
\text { values }\end{array}$ & 2.63 & 3.54 & 5.19 & 0.97 & 12.36 & 2.37 & 3.77 & 6.02 & 1.82 & 13.52 \\
\hline
\end{tabular}

$\mathrm{ChG}$ - chronic gastritis, $\mathrm{SChG}$ - superficial chronic gastritis. Glands categories: A - round; B - short oblique; C - long oblique; D - longitudinal. 
tively. In the statistical evaluation differences were not significant for these 3 types of glands. The results obtained from the automated image analysis (CAMI) and manual reading suggest high concordance for 3 types of glands: round shape, short oblique and long oblique. A difference between manual and automated counting of NE cells was noticed in the longitudinal glands: 0.97 and 1.82 , respectively. The results for the longitudinal glands should be verified in the extended study.

\section{Discussion}

The presented automated quantitative software (CAMI algorithm) based on sequential thresholding operation combined with the artificial neural network of Support Vector Machine (SVM) type [16,17]. This combined operation was applied in our previous studies on Index Ki-67 counted in neuroblastomas (immunopositive nuclear reaction) [18]. By introducing a complementary algorithm to this operation, the method was easily adapted for the image analysis of objects with cytoplasmic immunostaining as indicate our previous studies on bone marrow sections [19].

The CAMI algorithm extracts the individual cells (or the nuclei) from a background by detection of the objects stained more intensively than the less intensively stained surrounding. Extraction of the objects from the background has been explored by other researchers, however still the most common are methods based on one threshold value [12-15]. These approaches unnecessarily restrict possibilities of the cell separation and extraction. In our project the CAMI algorithm for glandular face reconstruction was based on the automated recognition of all intraglandular cells in the digital images, at 400x magnification, and involved several mathematical morphology operations: erosion, dilation, opening, closing, watershed method, skeletonisation, finding the cross points, reconstruction etc., that are implemented in Matlab [20,21].

Comparing our method with other studies of gastric image analysis we should mention two main methodological differences in the NE cells extraction and detection of the glands. In our study we used a sequential thresholding for the NE cells extraction as the most adequate extraction for nuclei from the image background. Such solution enables detect the "bright" cells from the background as much as they are stained more intensively than the rest of cells in the cluster. In other methods only a single threshold level was used (which is very restricted for cell extraction) or a solution based on "moved" window with a local threshold value (which is generally a very expensive solution) [13-15,22].

The automated detection of the separated glands is a difficult task. This problem has been studied in the histological models of the colitis or in prostatic sec- tions $[23,24]$. In our method we used the mask of cell nuclei as the start point for the gland detection. The reconstruction of the gland outlines was based directly on the gland face reconstruction. On the contrary to our principles the authors of another model proposed a detection of the gland lumen as the starting point of analysis. According to that method, the gland lumens were enlarged by a dilation operation and the obtained areas were acknowledged as the glands with the face cells. However this approach is restricted only to the glands with well visible lumen and omits a gland with invisible lumen. In one study the gland detection algorithm was based on the neighboring cells (for five nearest cells) [23]. Such approach is time consuming due to obligatory detection of every neighboring cell, separately. In both mentioned studies the image analysis was done in the slides stained with $\mathrm{H}+\mathrm{E}$ showing well visible continuous outlines of the glands. Our method is more adequate for the glands recognition with an discontinuous gland face which is much more difficult problem in some images e.g. in immunohistochemical staining. In the slides stained immunohistochemically, the contours of NE cells are unstained therefore their borders are not sharp nor well visible for the computerised recognition of the gland outlines. In light microscopy examination the recognition of the gastric gland outlines based on visible nuclei of gland cells and their spatial distribution. In the computerized gland recognition, the reconstruction of the glands shape must be done on the base of those sparsely lie nuclei. The second step of image analysis is recognition of immunopositive and immunonegative cells. The NE immunohistochemical staining gives the cytoplasmic immunoreaction on microscopic images, which is not always seen as an uniform perinuclear precipitant. Quantitative evaluation of the cell immunoreactivity is very subjective for the pathologists in light microscopy. It is also a significant problem for the computerized image analysis method which based on the analysis of a spatial distribution of immunostained cytoplasm and bordered nuclei.

It is worth to mention that the designed CAMI software for the digital reconstruction of the diversiform glands and for automated recognition and quantization of the intraglandular immunoreactive NE cells can be an eseful tool for the pathologists dealing with the digital quantitative pathology based on immunohistochemistry. The accuracy and feasibility of this new automated method for the quantitation of the immunoreactive cells within the glandular structure will be verify in our extended study.

Acknowledgements: This work was supported by grants of: the grant of Polish Ministry of Science and Higher Education for the years 2006-2009, the European Union in the framework of the European Social Fund through the Warsaw University of Technology Development Programme and the grant COST Action IC0604. 


\section{References}

[ 1] Bordi C, D'Adda T, Azzoni C, Ferraro G. Classification of gastric endocrine cells at light and electron microscopical levels. Microsc Res Tech. 2000;48:258-271.

[2] Falkmer S, Wilander E.The endocrine cell population. In: Whitehead R, ed. Gastrointestinal and oesophageal pathology. New York: Churcill Longman Group Ltd. 1995:63-71.

[3] Green DM, Bishop AE, Rindi G et al. Enterochromaffin-like cell populations in human fundic mucosa: quantitative studies of their variations with age, sex, and plasma gastrin levels. J Pathol. 1989;157:235-241.

[ 4] Tzaneva M, Julianov A. Chromogranin A-, somatostatin- and serotonin-containing endocrine cells in the corporal gastric mucosa of patients with Helicobacter pylori associated gastritis. Endocr Regul. 1999;33(2):79-82.

[ 5] Azzoni C, Doglioni C, Viale G, et al. Involvement of BCL-2 Oncoprotein in the Development of Enterochromaffin-like Cell Gastric Carcinoids. Am J Surg Pathol. 1996;20:433-41.

[6] Kozlowski W, Kulig A, Czkwianianc E et al. Morphological and immunohistochemical examinations of the dynamics changes of gastric mucosa associated with treatment of Helicobacter pylori infection in children. Ann Acad Medicae Bialostociensis. 1995;40(3):678-684.

[ 7] De Block CE, Colpin G, Thielemans K, Coopmans et al. Neuroendocrine tumor markers and enterochromaffin-like cell hyper/dysplasia in type 1 diabetes. Diabetes Care. 2004; 27(6):1387-93.

[ 8] Rindi G, Solcia E. Endocrine hyperplasia and dysplasia in the pathogenesis of gastrointestinal and pancreatic endocrine tumors. Gastroenterol Clin North Am. 2007;36(4):851-65.

[9] Aruin LG, Vinogradov VA, Zverkov JV, Nikiforov PA. The endocrine stomach cells containing endocrine gastrin and somatostatin in patients with duodenal ulcer. Arkhiv Patologii. 1984;XLVI(6):15-20.

[10] Yang L, Zhang HT, Zhang X, Sun YT, Cao Z, Su Q. Synchronous occurrence of carcinoid, signet-ring cell carcinoma and heterotopic pancreatic tissue in stomach: A case report and literature review. World J Gastroenterol. 2006;12(44): 7216-7220.

[11] Kasacka I, Majewski M. An immunohistochemical study of endocrine cells in the stomach of hyperinvasive rats. J Physiol Pharmacol. 2007;58(3):469-478.

[12] Bartels PH, Thompson D, Montironi R, Mariuzzi G, Hamilton PW. Automated reasoning system in histopathologic diag- nosis and prognosis of prostate cancer and its precursors. Eur Urol. 1996;30:222-233.

[13] Lezoray O, Elmoataz A, Cardot H, et al. Segmentation of color images from serous cytology for automated cell classification. Anal Quant Cytol. 2000;22:311-322.

[14] Kayser G, Radziszowski D, Bzdyl P, Sommer R, Kayser K. Theory and implementation of an electronic, automated measurement system for images obtained from immunohistochemically stained slides. Anal Quant Cytol. 2006;28:27-38.

[15] Kim YJ, Romeike BMF, Uszkoreit J, Feiden W. Automated nuclear segmentation in the determination of the Ki-67 labeling index in meningiomas. Clin Neuropathol. 2006;25(2):6773.

[16] Vapnik V. Statistical Learning Theory. New York: Wiley; 1998.

[17] Schölkopf B, Smola A. Learning with Kernels. Cambridge: MIT Press; 2002.

[18] Markiewicz T, Wisniewski P, Osowski S, Patera J, Kozlowski W, Koktysz R. Comparative analysis of the methods for accurate recognition of cells in the nuclei staining of the Ki67 in neuroblastoma and ER/PR status staining in breast cancer. Anal Quant Cytol. 2009;31:49-62.

[19] Markiewicz T, Osowski S, Patera J, Kozłowski W. Image processing for accurate recognition and counting of cells of the histological slides. Anal Quant Cytol. 2006;28:281-292.

[20] Soille P. Morphological image analysis, principles and application. Berlin: Springer; 2003.

[21] Matlab Image Processing Toolbox, user's guide. Natick: MathWorks; 2002.

[22] Kruk M, Osowski S, Koktysz R. Recognition and classification of colon cells applying the ensemble of classifiers. Comput Biol Med. 2009;39(2):156-165.

[23] Ficsor L, Varga VS, Tagscherer A, Tulassay Z, Molnar B. Automated classification of inflammation in colon histological sections based on digital microscopy and advanced image analysis. Cytom Part A. 2008;73A(3):230-237.

[24] Naik S, Doyle S, Agner S, Madabhushi A, Feldman M, Tomaszewski J. Automated gland and nuclei segmentation for grading of prostate and breast cancer histopathology. 5 International conference of biomedical imaging: From nano to macro, Paris. 2008:284-287.

Submitted:15 September, 2009 Accepted after reviews: 28 October, 2009 